Series A

\author{
I. MATHEMATICA
}

548

\title{
EXTENSION OF BOUNDARY HOMEOMORPHISMS OF DISCRETE GROUPS OF THE UNIT DISK
}

BY

PEKKA TUKIA

HELSIN K I 1973

S U O M A L A NEN TIEDEAKATEMIA

https://doi.org/10.5186/aasfm.1973.548 
Copyright $\mathbb{C} 1973$ by Academia Scientiarum Fennica ISBN 951-41-0119-7

Communicated 9 April 1973 by K. I. ViRTaxes 


\section{Extension of boundary homeomorphisms of discrete groups of the unit disk}

In this paper we consider discrete groups of the unit disk, i.e. groups whose elements are directly or indirectly conformal self-equivalences of the closed unit disk $E^{1}=\{z \in \mathbf{C}: z \leq 1\}$, acting discontinuously in the open unit disk $E=\{z \in \mathbf{C}:|z|<1\}$. If $F$ and $F^{\prime}$ are two such groups and $\varphi: F \rightarrow F^{\prime}$ an isomorphism, it is said to be geometric if there is a homeomorphism $f$ of the open unit disk $E$ such that (1) below is true.

$$
f(T(x))=q(T)(f(x)) \text { for } x \in E \text { and } T \in F .
$$

In this case we say that $f$ induces $q$.

If $T$ is a hyperbolic transformation of the unit disk we denote by $\operatorname{Ax}(T)$ the hyperbolic line joining the attractive and the repelling fixed point of $T$ including the endpoints. It $T$ is parabolic, $A x(T)$ is also defined; it consists of one point only, of the fixed point of $T$. Then (2) below is the axis condition for $\varphi$. It is always fulfilled if $\varphi$ is geometric.

(2) $\mathrm{Ax}(T)$ and $\mathrm{Ax}(S)$ intersect if and only if $\mathrm{Ax}(\varphi(T))$ and $\mathrm{Ax}(\varphi(S))$ intersect, $T, S \in F$ hyperbolic or parabolic.

We shall show that condition (2) implies the existence of a homeomorphism of $E$ inducing $\varphi$ if $F$ is a group whose limit set contains more than two points, i.e. if $F$ contains a free subgroup with two generators (cf. p. 15). This is previously known for a large class of groups (cf. Tukia [4] pp. 32-33) including those not containing reflections.

It was also shown in [4] (pp. 31-32) that if $F$ and $F^{\prime}$ are groups of the first kind and $f$ is a homeomorphism of $E$ inducing $\varphi$, then $f$ admits a continuous extension to a homeomorphism $g$ of the closed unit disk $E^{1}$. The restriction $h=g: S^{1}$ of $g$ to the unit circle $S^{1}=$ $\{z \in \mathbf{C}:|z|=1\}$ is a homeomorphism of $S^{1}$ such that the following is true.

$$
h(T(x))=q(T)(h(x)) \text { for } x \in S^{1} \text { and } T \in F .
$$

In general if $\varphi: F \rightarrow F^{\prime}$ is an isomorphism of discrete groups of the unit disk, not necessarily of the first kind, a homeomorphism $h: S^{1} \rightarrow S^{1}$ of the unit circle is called a boundary homeomorphism of $F$ and $F^{\prime}$ in- 
ducing $\varphi$ if it fulfils (3). We remark that $h$ is uniquely determined by $\varphi$ only if $F$ and $F^{\prime}$ are of the first kind.

The existence of a boundary homeomorphism inducing of and the axis condition (2) for $\varphi$ are equivalent if the groups are of the first kind but this is not true in general. The axis condition is weaker and is implied by the existence of a boundary homeomorphism. Again, as we have shown above, with Fuchsian groups of the first kind, a boundary homeomorphism can always be extended to a homeomorphism of the closed unit disk $E^{1}$ that induces $\varphi$. Our second result will be (cf. theorem 3 p. 12 later) that this is true also for groups of the second kind.

We shall use notation of [4] (cf. especially section 1.A). All the references, too, are to [4] except when otherwise stated. We remark that it is not possible to read this paper without the knowledge of [4].

The types of boundary of a pointed surface. Let $F$ be a discrete group of the unit disk. We denote by

$$
\lim (F)
$$

the set of limit points of $F$ and by

$$
L(F)
$$

the union of $\lim (F)$ and the endpoints of the axes of reflections of $F$.

In [4] we assumed that a discrete group $F$ of the unit disk acted in the open unit disk $E$ and the quotient space $S$ was $E / F$. As a consequence $S$ had a boundary if and only if $F$ had reflections. In the present case we allow for the possibility of $F$ acting in a slightly larger space $E^{\prime}$ where $E \subset E^{\prime} \subset E^{1} \backslash L(F)$ and $E^{\prime} \cap S^{1}$ is open in $S^{1}$. When we say that $F$ acts in $E^{\prime}$ we always mean that $F$ acts discontinuously in $E^{\prime}$. Thus the quotient space $E^{\prime} / F$ has two kinds of boundary points: those that belong to $\left(E^{\prime} \backslash E\right) / F$ and the quotient sets of points $x$ in $E$ that are on the axes of reflections of $F$. If we have no knowledge about the group $F$, we cannot, as a rule (cf. theorem 2 later), know whether a boundary point is in the set $\left(E^{\prime} \backslash E\right) / F$ or not. It is perfectly. possible to have homeomorphic pointed surfaces $S=E / F$ and $S^{\prime}=E^{\prime} \mid F$ where $F$ has reflections and $F^{\prime}$ not. That is why we divide the boundary of $S$ into two distinct sets $B_{1}$ and $B_{2}$ where $B_{1}$ originates from the reflections of $F$ and $B_{2}$ is the quotient set $\left(E^{\prime} \backslash E\right) / F$. We call $B_{1}$ the reflective boundary of $S$ and $B_{2}$ the ordinary boundary of $S$. Thus the group $F$ determines a pointed surface denoted $\left(S, B_{2}, n\right)=$ $\left(S_{F}, B_{F}, n_{F}\right)$ (cf. the definition of $\left(S_{F}, n_{F}\right)$ on p1) 10-11 in [4] when $F$ acts in $E$ ). 
Thus we are led to consider triples of the form $(S, B, n)$ where $S$ is a countable bordered surface, $B$ a union of the components of the boundary $\partial S$ of $S$ and $n: S \rightarrow \mathbf{N}$ a mapping with discrete support $n=\{x: n(x)>1\}$ disjoint from $B$. The surface $S$ may or may not have a conformal structure. Given such a triple $(S, B, n)$ we ask if there exists a discrete group $F$ of the unit disk acting in $E^{\prime}, E \subset E^{\prime} \subset$ $E \backslash L(F)$ such that $(S, B, n)=\left(E^{\prime} / F,\left(E^{\prime} \backslash E\right) / F, n_{F}\right)$ where $n_{F}(\mathrm{cl} x)$ depends on the stabilizer of $x$ (cf. [4] pp. 10-11). This can be answered casily by considerations of [4]. We remark that the analogies of proposition 2.4 and corollaries 2.4.1, 2.4.2 and 2.4.3 in [4] hold also true for pointed surfaces of the form $(S, B, n)$. These together with theorem 2.8 show that if $B \neq \emptyset,(S, B, n)$ has a representation of the desired form. This can be seen as follows. We first consider the triple $\left(s^{\prime} \backslash B, \varnothing, n\right)$, $s$ endowed with some conformal structure of a bordered Riemann surface. The results mentioned above show that this triple can be represented in the form $\left(s_{F}, \sigma, n_{F}\right)$ tor some discrete group of the unit disk. After that we simply add suitably intervals of $S^{1}$ to $E$ to form the space $E^{\prime}$ such that $E^{\prime} / F$ is conformally equivalent to $S$.

to far we have not considered the set $E^{1} \backslash E^{\prime}$. It is clear that it may differ from the set $L(F)$. When starting with a given discrete group $F$ of the unit disk it is most natural to consider the triple of the form $\left(S_{F}, B_{F}, n_{F}\right)$ where $F$ acts in $E^{\top} \backslash L(F)$. We ask now, given a pointed surface $(S, B, n)$, if it can be represented in this form if we do not demand conformal equivalence of the pointed surfaces $(S, B, n)$ and $\left(S_{F}, B_{F}, n_{F}\right)$. Simple examples of the form $\left(E^{\prime}, E^{\prime} \backslash E, 1\right)$ where $E \subset E^{\prime} \subset E^{1}$ and $E^{\prime}$ is open in $E^{1}$ show that this is not always possible. But later we shall show that we can demand that $F$ acts in $E^{\prime}$ where $\mathrm{d}\left(E^{\prime} \cap S^{1}\right)=S^{1}$, supposing $B=\varnothing$.

The technique for proving the following theorems is basically the same ws that used in [4]. Thus we first consider representations of pointed surfaces.

Representations for pointed surfaces. Let $(S, B, n)$ be a pointed surface such that all boundary components of $S$ are included in $B$, i.e. $B=\partial S$. A representation of $(S, B, n)$ is defined as follows. Let $A$ be a plane domain bounded by a Jordan curve $J$. Let $E^{\prime}$ be a subset of $A \cup J$ with $A \subset E^{\prime}$. Further, we suppose that two sets of intervals of $J$ are given; let these be $\left\{I_{i}\right\}, i \in K$, and $\left\{I_{j}\right\}, j \in L,(K \cap L=\varnothing)$. The intervals may be open, half-open or closed (but not points) and their union will be $E^{\prime} \cap J$. We require that:

(i) $I_{i} \cap I_{j}$ is either empty or is a common endpoint, $i \neq j, i$, $j \in K \cup L$. 
(ii) $I_{k}, k \in K$, is a component of $\left(E^{\prime} \cap J\right) \backslash \bigcup_{i \in L} I_{i}$ when its (possible) endpoints are removed.

(iii) If $x$ is an endpoint of $I_{i}, i \in L, x \in I_{i}$, then it is an endpoint for another $I_{j}, j \in K \cup L, x \in I_{j}$.

(iv) $I_{i}, i \in L$, intersects with at most one $I_{j}, j ; i, j \in L$.

The set of vertices is

$$
V=U\left(I_{i} \cap I_{j}\right) \cdot i \quad j, i \cdot j \in I .
$$

Let

$$
i: \bigcup_{i \in L} I_{i} \rightarrow \bigcup_{i \in I} I_{i}
$$

be a homeomorphism such that

$$
\begin{aligned}
& i^{2}=i d, \\
& i\left(I_{i}\right)=I_{j} \text { for another } j \in I,(j ; i) \text { when } i \in L . \\
& i V V=i d .
\end{aligned}
$$

Let $S^{\prime}$ be the surface obtained from $E^{\prime}$ by identifying and $i(x)$ for $x \in \bigcup_{i \in L} I_{i}$. If there is a homeomorphism $f: s^{\prime \prime} \rightarrow s$ such that $|n|=f(k(V))$ when $k: E^{\prime} \rightarrow S^{\prime}$ is the canonical projection, we say that $\left(E^{\prime}, i, f\right)$ is a representation for $(S, B, n)$. We remark that the intervals $I_{i}, i \in K \cup L$, as well as the sets $\left\{I_{i}\right\}, i \in L$ and $\left\{I_{j}\right\}, j \in K$, are uniquely determined by $\left(E^{\prime}, i, f\right)$.

Theorem 1. Let $(S, B, n)$ be a pointed surface such that $B$ is the boundary of $S$ and $S$ non-compact or $B=0$. Then there is a representation $\left(E^{\prime}, i, f\right)$ of $(S, B, n)$ where $E \subset E^{\prime} \subset E^{1} . E^{\prime}$ is open in $E^{1}$, and such that any two intervals identified by i hare equal lenglh. If the pointed surface $(S, B, n)$ is not equivalent to $(E .0 .1)$ we may besides assume that $E^{\prime} \cap S^{1}$ is dense in $S^{1}$.

We shall suppose that $B$ o for otherwise theorem 1 is a consequence of proposition $2.7 \mathrm{in}$ [4]. Te can also assume that $s$ is not compact for in this case theorem 1 is easily secn to be true by using the classification theorems for compact bordered surfaces. Wo shall consider sequences $K_{1} \subset K_{2} \subset \ldots$ satisfying the following conclitions.

(i) $S=\bigcup_{i=1}^{\infty} K_{i}$

(ii) Each $K_{i}, i \geq 1$, is a compact bordered subsuiface of 's such that $K_{i} \cap \partial S$ is a finite union of .Jordan ares or Jordan curves of $\partial S$. 
(iii) $K_{i} \subset$ int $K_{i+1}, i \geq 1$.

(iv) The support of $n$ does not intersect with the boundary of $K_{i}, i \geq 1$.

(v) Each component of $\operatorname{cl}\left(S \backslash K_{i}\right)$ is non-compact, $i \geq 1$.

(vi) Each component of $\operatorname{cl}\left(S \backslash K_{i}\right)$ that does not intersect with $B$ has in common with $K_{i}$ exactly one Jordan curve.

(vii) If $\left('\right.$ is a component of $\operatorname{cl}\left(S \backslash K_{i}\right)$ intersecting with $B$ then each component of $C \cap K_{i}$ is a Jordan arc whose endpoints lie on $B$.

An exhaustion fulfillng conditions (i)-(vii) is called a canonical exhaustion of $(S, B, n)$. We should verify that such exhaustions exist. The construction method is that of Ahlfors-Sario [1] ch. 1 section 29 for non-compact surfaces without boundary. It is easy to see that there exist exhaustions satisfying (i)-(v). The condition (vi) is then added like in Ahlfors-Sario ch. 129 C. (If a component $C$ of $\operatorname{cl}\left(S \backslash K_{i}\right.$ ) has two or more Jordan curves in common with $K_{i}$ their number can be reduced as follows. Let $J_{1}$ and $J_{2}$ be two Jordan curves in $C \cap K_{i}$. Connect them with a Jorlan are $J$ with one endpoint in $J_{1}$ and the other in $J_{2}$, all inner points of $J$ lying in $S \backslash K_{i}$. Now $J$ is slightly enlargened to a strip $A$ whose boundary in $\operatorname{cl}\left(S \backslash K_{i}\right)$ together with subarcs of $J_{1}$ and $J_{2}$ form a new Jordan curve $J^{\prime}$. Now $K_{i} \cup A$ has fewer Jordan curves in common with $\operatorname{cl}\left(S \backslash\left(K_{i} \cup A\right)\right)$ than $\left.K_{i}\right)$. Similarly, if an exhaustion $K_{1}, K_{2} \ldots$ does not satisfy (vii) we can proceed as follows. Let $C, C \cap B=\varnothing$, be a component of $\operatorname{cl}\left(S \backslash K_{i}\right)$ which contains a Jordan curve $J$ not intersecting with $B$. We connect $J$ by a Jordan are to $B$. Then one endpoint of $J^{\prime}$ lies on $B$, the other lies on $J$ and the inner points belong to $S \backslash K_{i}$. This arc $J^{\prime}$ is slightly enlargened to a strip $A$ whose boundary in $S \backslash K_{i}$ together with a subare of $J$ is a Jordan arc whose endpoints lie on $B$. The number of Jordan curves of $\operatorname{cl}\left(S \backslash\left(K_{i} \cup A\right)\right)$ in common with $K_{i} \cup A$ is smaller than the number of Jordan curves of $\operatorname{cl}\left(S \backslash K_{i}\right)$ that are in common with $K_{i}$.

It is a consequence of (vii) that each component of $\operatorname{bd}\left(K_{i}\right), i \geq 1$, intersects with $B$ unless it is contained in a component of $\operatorname{cl}\left(S \backslash K_{i}\right)$ whose intersection with $B$ is empty. In particular there are ares of $b d\left(K_{1}\right)$ that intersect with $B$, since $B$ is assumed to be non-empty.

We have in [4] treated the case of $(S, B, n)$ with $B \neq \varnothing$. (Cf. proposition 2.7 p. 21). To avoid repeating the argumentation used there we define a new exhaustion $K_{1}^{\prime} \subset K_{2}^{\prime} \subset \ldots$ of $(S, B, n)$ as well as formulate a lemma that is the form in which we use proposition 2.7.

We set

$$
\begin{aligned}
& K_{1}^{\prime}=K_{1} \\
& K_{i}^{\prime}=K_{i} \cup\left(\bigcup_{j \in I_{j}} S_{j}\right), i>1 .
\end{aligned}
$$


where $S_{j}, j \in I_{i}$, are components of $\operatorname{cl}\left(S \backslash K_{i}\right)$ that do not intersect with $B$.

Lemma 1. Let $C$ be a circle arc orthogonal to $S^{1}$ in $E^{1}$ and let $E^{\prime}$ be the closure of a component of $E^{1} \backslash C$. Let $(S, B, n)$ be a pointed surface with $B$ homeomorphic to $S^{1}$. Let $g: C /\{x, y\} \rightarrow B$ be a homeomorphism where $x$ and $y$ are the endpoints of $C$. Then there is a representation $\left(E^{\prime \prime}, i, f\right)$ of $(S, B, n)$ with $g=f \circ k \mid C$ when $k$ is the canonical projection $k: E^{\prime \prime} \rightarrow E^{\prime \prime} / i$, cl $E^{\prime \prime}=E^{\prime}$ and $\operatorname{cl}\left(E^{\prime \prime} \cap S^{1}\right)=E^{\prime} \cap S^{1}$.

The validity of the above lemma follows from proposition 2.7. For, according to this proposition, $(S, B, n)$ has a representation $\left(E_{1}, i, f\right)$ where cl $E_{1}$ is homeomorphic to a closed disk from which an open disk has been removed and the boundary of this removed disk corresponds to $B$.

After that we begin the construction of the representation for $(S, B, n)$. This is done step by step constructing first a representation $\left(E_{j}, i_{j}, f_{j}, S_{j}, k_{j}\right)$ for $\left(K_{j}^{\prime}, \partial K_{j}^{\prime}, n \mid K_{j}^{\prime}\right), j \geq 1$, where we have for clarity written the triple $\left(E_{j}, i_{j}, f_{j}\right)$ as a quintuple and where $S_{j}=E_{j} / i_{j}$ and $k_{j}$ the canonical projection $E_{j} \rightarrow S_{j}$. As we have already observed $K_{1}^{\prime} \cap B \neq \varnothing$. Using this result and the classification theorems for compact bordered surfaces we see that there is a representation $\left(E_{1}, i_{1}, f_{1}\right.$, $\left.S_{1}, k_{1}\right)$ for $\left(K_{1}, \partial K_{1}, n: K_{1}\right)$ satisfying the following conditions.

(i) $E_{1}$ is a closed Jordan domain bounded by $S^{1}$ and a set of disjoint closed circle arcs orthogonal to $S^{1}$.

(ii) $k_{1}^{-1} f_{1}^{-1}\left(K_{1} \cap B\right)$ is a set of disjoint closed intervals of $S^{1}$.

(iii) $k_{1}^{-1} f_{1}^{-1}\left(\partial K_{1} \backslash B\right)$ is a disjoint set of open or closed circle arcs orthogonal to $S^{1}$ whose endpoints belong to $k_{1}^{-1} f_{1}^{-1}(B)$ unless its endpoints are identified by $i_{1}$.

(iv) The intervals identified by $i_{1}$ are either closed circle arcs orthogonal to $S^{1}$ whose endpoints belong to $k_{1}^{-1} f_{1}^{-1}(B)$ or closed intervals of $S^{1}$ at least one endpoint of which belongs to $k_{1}^{-1} f_{1}^{-1}(B)$. The other endpoint if it does not belong to $k_{1}^{-1} f_{1}^{-1}(B)$ belongs either to the set of vertices of the representation (to the set $V p .6$ ) or is an endpoint of the circle arc orthogonal to $S^{1}$ whose endpoints are identified by $i_{1}$.

After that we extend $\left(E_{1}, i_{1}, f_{1}, S_{1}, k_{1}\right)$ to a representation of $\left(K^{\prime}, \partial K^{\prime}, n \mid K^{\prime}\right)$ where $K^{\prime}$ is $K_{1}$ added by a component $K^{\prime \prime}$ of $\operatorname{cl}\left(K_{2}^{\prime} \backslash K_{1}\right)$. We first consider the case in which this component is compact. We then construct a representation $\left(E^{\prime \prime}, i^{\prime \prime}, f^{\prime \prime}, S^{\prime \prime}, k^{\prime \prime}\right)$ of $\left(K^{\prime \prime}, \partial K^{\prime \prime}, n \mid K^{\prime \prime}\right)$ satisfying conditions similar to (i)--(iv). Besides we 
may assume that $E_{1} \cap E^{\prime \prime}$ is a circle arc orthogonal to $S^{1}$ and that $f_{1} k_{1}\left|E_{1} \cap E^{\prime \prime}=f^{\prime \prime} k^{\prime \prime}\right| E_{1} \cap E^{\prime \prime}$. Now we define the representation $\left(E^{\prime}\right.$, $i^{\prime}, f^{\prime}, S^{\prime}, k^{\prime}$ ) with $E^{\prime}=E_{1} \cup E^{\prime \prime}$ where we set

$$
\begin{aligned}
& i^{\prime} \text { domain of } i_{1}=i_{1}, \\
& i^{\prime} \text { domain of } i^{\prime \prime}=i^{\prime \prime} .
\end{aligned}
$$

Besides there may be sets of circle arcs $I_{k} \subset E_{1}$ and $J_{k} \subset E^{\prime \prime}, k \in N$, orthogonal to $S^{1}$ such that $f_{1} k_{1}\left(I_{k}\right)=f^{\prime \prime} k^{\prime \prime}\left(J_{k}\right)$. Then we define $i^{\prime}$ on $\bigcup_{k \in N}\left(I_{k} \cup J_{k}\right)$ in such a way that the resulting quotient space is homeomorphic to $K^{\prime}$ by a homeomorphism $f^{\prime}$.

If the component $K^{\prime \prime}$ added to $K_{1}$ is not compact we obtain by the above lemma 1 a representation for $K^{\prime \prime}$. Other details are similar and are omitted. There are only a finite number of components to be added to $K_{1}$ to obtain $K_{2}$, so after a finite number of steps we have a representation $\left(E_{2}, i_{2}, f_{2}, S_{2}, k_{2}\right)$ for $\left(K_{2}^{\prime}, \partial K_{2}^{\prime}, n \mid K_{2}^{\prime}\right)$. It should be remarked that this representation fulfils conditions similar to (i)-(iv) except that we must remember that non-compact components may be added to $K_{1}$. Therefore we add to conditions (i) - (iv) the condition (v). Let $I$ and $J$ be two intervals of $S^{1}$ identified by $i_{2}$. Then $I$ may also be open or half-open.

(v) If $I$ is half-open, the endpoint belonging to it is rither a vertice of the representation or belongs to the set $k_{2}^{-1} f_{2}^{-1}(B)$.

The above discussion shows that we can form a series of representations $\left(E_{n}, i_{n}, f_{n}, S_{n}, k_{n}\right)$ for $\left(K_{n}^{\prime}, \partial K_{n}^{\prime}, n, K_{n}^{\prime}\right), n \geq 1$. so that conditions similar to $(\mathrm{i})-(\mathrm{v})$ hold true and that for $n \geq 2$

$E_{n-1} \subset E_{n}$

$i_{n} \mid$ domain of $i_{n-1}=i_{n-1}$,

$f_{n} \mid S_{n-1}=f_{n-1}$.

Besides we may demand:

(vi) The diameter of components of $E_{n} \quad E_{n-1}<1 n . " \geq 2$.

Then the representation $\left(E^{\prime}, i, f\right)$ is defined as follows.

$$
E^{\prime}=\bigcup_{j=1}^{\infty} E_{j}
$$

domain of $i=\bigcup_{j=1}^{\infty}$ domain of $i_{j}$,

$i \mid$ domain of $i_{j}=i_{j}, j \geq 1$.

$f: S_{j}=f_{j}, j \geq 1$. 
The triple is not yet the representation we have sought. First of all it may be that $E \notin E^{\prime}$. This is remedied by substituting for every circle arc orthogonal to $S^{\mathbf{1}}$ belonging to the boundary of $E^{\prime}$ the corresponding arc of $S^{1}$ and modifying $i$ suitably. We suppose this is done. After that $\operatorname{cl}\left(E^{\prime} \cap S^{1}\right)=S^{1}$ but two intervals identified by $i$ may not have equal length. However, by the following lemma 2 , the representation $(E, i, f)$ can be replaced by another that has this property.

Lemma 2. Let $\left(E^{\prime}, i, F\right), E \subset E^{\prime} \subset E^{1}$, be a representation for some pointed surface $(S, B, n)$. Then there is a homeomorphism $f$ of $S^{1}$ such that if $I$ and $J$ are two intervals identified by $i$ then $f(I)$ and $f(J)$ have equal lengih.

Proof: Let $V$ be the set of vertices of the representation and let $I_{k}, J_{k}$, $k \in k, \quad i\left(I_{k}\right)=J_{k}$, be the intervals of $E^{\prime} \cap S^{1} \backslash V$ identified by $i . W^{\mathrm{e}}$ choose a $k \in K$ and define a homeomorphism $f_{k}: S^{1} \rightarrow S^{1}$ as follows. Fo simplicity we assume that the endpoints of $I_{k}$ are $e^{\circ}$ and $e^{x i}$ and those of $J_{k} e^{\pi i}$ and $e^{(x-y) i}, o<x \leq(\pi-y)<\pi$. Let $f_{k}=i d$ restricted to the lower half of $y \leq o$ of the circle. Let $f_{k} \mid(\exp o, \exp x i)$ be the linear stretch to the interval $(\exp o, \exp (x+y) i \cdot 2), f_{k} \mid(\exp (\pi-y) i, \exp \pi i)$ be the linear stretch to the interval $(\exp (\tau-(x+y) / 2) i, \exp \pi i)$ and let $f_{k}$ restricted to $(\exp x i, \exp (\pi-y) i)$ be the rotation to the interval $(\exp (x+y) i 2 \exp (\tau-(x+y) / 2) i)$. We remark that $f_{k}$ does not change the lengths of the other intervals $I_{l}, J_{l}, k \neq l, l \in K$, and that the difference between arc $x$ and arc $f_{k}(x)$ is not more than $1 / 2 /$ length of $I_{k}-$ length of $J_{k}$.

We shall denote this mapping by $f_{1}$. After that we choose another index $l \in K$ and define the mapping $f_{2}$ in the same manner with respect to the intervals $f_{1}\left(I_{l}\right)$ and $f_{1}\left(J_{l}\right)$. We continue in this way. If $K$ is finite. $K=\left\{k_{1} \ldots k_{n}\right\}$ :

$$
g_{n}=f_{n} O \ldots o f_{1} .
$$

will be the required homeomorphism. Otherwise there will be a limit $\lim _{n \rightarrow \infty} g_{n}$ that is the required homeomorphism.

Theorem 2. Let $(S, B, n)$ be a pointed surface such that $S \backslash B$ is noncompact. Then it can be represented, up to a homeomorphism, in the form $\left(S_{F}, B_{F}, n_{F}\right)$ by means of a discrete group $F$ of the unit disk acting in a set $E^{\prime}, E \subset E^{\prime} \subset E^{1} \backslash L(F), E^{\prime}$ open in $E^{1}$. Moreover, we can assume that either $E^{\prime} \cap S^{1}=\varnothing$ or $\operatorname{cl}\left(E^{\prime} \cap S^{1}\right)=S^{1}$. If $\lim F$ contains more than two points, $\lim F=S^{1}$ if $B=\varnothing$. In addition, if $\partial S=B$, i.e. $F$ does not contain reflections we may assume that $F$ is formed as a free combination of cyclic groups. 
In case $B=\partial S$, the proof of theorem 2 is quite straightforward, once we have theorem 1 . It is a repetition of the arguments used to prove theorem 2.8 p. 25 in [4]. We shall not do it here again. Only the case with $\lim F$ containing at most two points requires a comment. But before we do this we treat the case with non-empty reflective boundary.

Let $(S, B, n)$ be a pointed surface with $\partial S \neq B$. We represent the triple $(S, \partial S, n \mid$ int $S)$ by means of a discrete group $F^{\prime \prime}$ of the unit disk acting in $E^{\prime}, E \subset E^{\prime} \subset E^{1}, E^{\prime}$ open in $E^{1}$, where $\operatorname{cl}\left(E^{\prime} \cap S^{1}\right)=S^{1}$. The conformal structure of $S$ is defined to be equal with that of $S_{F^{\prime \prime}}=$ $E^{\prime} / F^{\prime \prime}$. This can be done since we know that $\partial S$ is non-empty. Let $B_{1}=$ $p^{-1}(B)$ when $p: E^{\prime} \rightarrow S$ is the canonical projection. Define

$$
S^{*}=\left(E^{\prime}\left\lfloor E^{\prime}\right) /\left(E^{\prime} \cap S^{1} \backslash B_{1}\right)\right.
$$

i.e. we take two copies of $E^{\prime}$ and identify the boundary points $x$ for which $p(x) \in \partial S \backslash B$. This is a bordered surface whose boundary consists of two copies of $B_{1}$ denoted $B^{*}=B_{1} \perp B_{1}$. A mapping $n^{*}: S^{*} \rightarrow \mathbf{N}$ is defined by setting $n^{*}(x)=n\left(p\left(p^{*}(x)\right)\right.$ if $x \in E^{\prime} \cap S^{1}$ and $=1$ otherwise when $p^{*}: S^{*} \rightarrow E^{\prime}$ is the canonical projection. Since either $B^{*} \neq \varnothing$ or $S^{*}$ is non-compact, there is by the first part of the proof of theorem 2 a discrete group $F^{\prime}$ acting in $E^{\prime \prime}, E \subset E^{\prime \prime} \subset E^{1} \backslash L\left(F^{\prime}\right)$ such that $\left(S_{F^{\prime}}, B_{F^{\prime}}, n_{F^{\prime}}\right)$ $=\left(S^{*}, B^{*}, n^{*}\right)$. We denote the projection $E^{\prime \prime} \rightarrow S^{*}$ by $p^{\prime}$. It may be that the combined projection $p_{1}=p o p^{*} o p^{\prime}$ is not a local conformal equivalence at points of $p_{1}^{-1}(|n| \cup(\partial S \backslash B))$ but the conformal structure of $E^{\prime \prime}$ can be redefined so that it is. Then the homeomorphisms $f$ of $E^{\prime \prime}$ for which the triangle

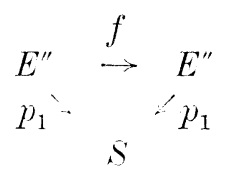

commutes, form by analogy of corollary 2.4.1 in [t] for the bordered case a group $F$ of conformal self-equivalences of $E^{\prime \prime}$ so that $\left(S_{F}, B_{F}, n_{F}\right)$ is equivalent, up to a homeomorphism, to $(S, B, n)$.

If $F$ contains a free subgroup with two generators then $E^{\prime \prime}$ minus its boundary is conformally equivalent to $E$. So we may assume that it is $E$ added with a union of intervals of $S^{1}$. Moreover it is seen that $E^{\prime \prime} \cap S^{1}$ is empty if and only if $\lim F$ is dense in $S^{1}$, for the emptiness of $E^{\prime \prime} \cap S^{1}$ is equivalent to the emptiness of $B_{1}$ and thus with the emptiness of $B^{*}$ and $B$. If $\lim F$ is not dense in $S^{1}$ we see that $E^{\prime \prime} \cap S^{1}=$ $p^{*-1}\left(B^{*}\right)=p_{1}^{-1}(B)$ is dense in $S^{1}$.

After that we treat the case in which $F$ does not contain a free subgroup with two generators. If $B=\partial S$, then the discussion in theorem 2.8 p. 25 in [4] shows that this can happen if and only if $\mid n=\alpha$ and 
$S \backslash B$ is a sphere punctured in two points or a projective plane punctured in one point or of the type $(E, \varnothing, n)$ where $n \mid=x$ or $n=\{x, y\}$ with $n(x)=n(y)=2$. Suppose then that $\partial S \backslash B \neq \varnothing$. If $|n|=\varnothing$ we see that $(S \backslash B, \varnothing, n)$ is homeomorphic either to the complex plane with the open unit disk removed or with the $\operatorname{strip}\{z \in \mathbf{C}: 0 \leq y \leq 1\}$. If $|n| \neq \varnothing,(S \backslash B, \varnothing, n)$ is homeomorphic to the half plane $\{z \in \mathbf{C}$ : $y \geq o\}$ with $|n|=\{0\}$ or $|n|=\{i\}$ and $n(i)=2$ or $n \mid=\{-1,1\}$ with $n(-1)=n(1)=2$. In any case we see that the terms of the theorem can be met.

The boundary homeomorphism. In this section we discuss the case of a boundary homeomorphism inducing an isomorphism $\psi: F \rightarrow F^{\prime}$ between two discrete groups of the unit disk. First we formulate a lemma for use in theorem 3 .

Lemma 3. Let $\varphi, F$ and $F^{\prime}$ be as above and suppose that $F$ acts in $E^{\prime}, E \subset E^{\prime} \subset E^{1}, E^{\prime}$ open in $E^{1}$ and that $F^{\prime}$ acts in $E^{\prime \prime}$, with similar properties. If $\operatorname{cl}\left(E^{\prime} \cap S^{1}\right)=S^{1}=\operatorname{cl}\left(E^{\prime \prime} \cap S^{1}\right)$ or both groups are of the first kind and if $f: E^{\prime} \rightarrow E^{\prime \prime}$ is a homeomorphism such that

$$
f(T(x))=\varphi(T)(f(x)) \text { for } \quad x \in E^{\prime} \text { and } T \in F
$$

then $f$ can be extended to a homeomorphism $g: E^{1} \rightarrow E^{1}$ such that (*) is valid for all $x \in E^{1}, T \in F$ if $f$ is replaced $g$.

Proof: If $F$ and $F^{\prime}$ are of the second kind, proof is obvious. If they are of the first kind see [4] pp. $31-32$.

Theorem 3. Let $F, F^{\prime}$ and $q$ be as above and suppose that there exists a boundary homeomorphism $h: S^{1} \rightarrow S^{1}$ satisfying

$$
h(T(x))=\varphi(T)(h(x)) \text { for } x \in S^{1} \text { and } T \in F .
$$

Then there exists a homeomorphism $f: E^{1} \rightarrow E^{1}$ extending $h$ such that (1) is valid for all $x \in E^{1}$ and $T \in F$, h replaced by $f$.

Proof: We assume that $E / F$ is non-compact. For $E F$ compact see references [2]-[5]. In this case any isomorphism if is incluced by a homeomorphism $f: E \rightarrow E$ that can be extended to $E^{1}$ ([4] p. 32).

We first assume that $F$ and consequently $F^{\prime}$ does not contain reflections. Let $\left(S_{F}, B_{F}, n_{F}\right)$ be the pointed surface defined by $F$, acting in $E^{\prime} \backslash L(F)$. By theorem $2\left(S_{F}, B_{F}, n_{F}\right)$ is up to a homeomorphism equivalent to a pointed surface $\left(S_{G}, B_{G}, n_{G}\right)$ where $G$ is either of the first kind or it acts in $E^{\prime}, E \subset E^{\prime} \subset E^{1}, E^{\prime}$ open in $E^{1}$, and that $\operatorname{cl}\left(E^{\prime} \cap S^{1}\right)=S^{1}$ and that it is formed as a free combination of cyclic 
groups. In both cases by corollary 2.4.3 in [4] for bordered surfaces there exists a homeomorphism $g: E^{\prime} \rightarrow E^{1} \backslash L(F)$ such that

$$
g(T(x))=\varphi^{\prime}(T)\left(g(x) \text { for } x \in E^{\prime} \text { and } T \in G\right.
$$

(if $F$ is of the first kind $E^{\prime}=E$ ) where $\varphi^{\prime}: G \rightarrow F$ is an isomorphism. By lemma $3 \mathrm{~g}$ can be extended to a homeomorphism $E^{1} \rightarrow E^{1}$ so that (2) is satisfied by all $x \in E^{1}$ and $T \in G$. Thus if we replace $F$ by $G$, $\varphi$ by $\varphi \circ \varphi^{\prime}$ and the boundary homeomorphism $h$ by $h \circ g \mid S^{1}$ we may assume that the group in question is formed as a free combination of cyclic groups. We suppose this is done.

Let $F_{i}, D_{i}, T_{i}, i \in I$, form $F$ as a free combination of cyclic groups where these notations are used as in p. 16 of [4]. Then we define $F_{i}^{\prime}, D_{i}^{\prime}$, $T_{i}^{\prime}, i \in I$, by means of the boundary homeomorphism $h$ as was done on pp. 34-35 of [4]. The proof is complete if we can show that $F_{i}^{\prime}, D_{i}^{\prime}$, $T_{i}^{\prime}, i \in I$, form indeed $F^{\prime}$ as a free combination of cyclic groups. (The details of defining the homeomorphism inducing $\varphi$ can then be found on p. 35 of [4] and can be readily modified to the present case.) According to the proof of theorem $2.6 \mathrm{in}$. [4] this is equivalent with the fact that $D^{\prime}$ below is the fundamental domain for $F^{\prime}$. Let

$$
\begin{aligned}
& D^{\prime}=\bigcap_{i \in I} D_{i}^{\prime} \\
& D^{\prime \prime}=\bigcup_{T \in F^{\prime}} T\left(D^{\prime}\right) .
\end{aligned}
$$

By theorem 2.6, $D^{\prime}$ is the fundamental domain for $F^{\prime}$ if and only if $D^{\prime \prime}$ is the whole open unit disk. Assume the contrary and let $x$ be an element of $E \backslash D^{\prime \prime}$. Then define a chain $\left\{D_{n}^{\prime}\right\}, n \geq 0$, by (i) and (ii) p. 18 in [4]. Let $\left\{D_{n}\right\}, n \geq o$, be the chain defined by $D_{n}=T_{n}(D)$ if $D_{n}^{\prime}=$ $\varphi\left(T_{n}\right)\left(D^{\prime}\right)$ and $D=\bigcap_{i \in I} D_{i}, n \geq o$. Since $D$ is a fundamental domain for $F$ this chain converges towards a point $y$ of $S^{1}$. But then $\left\{D_{n}^{\prime}\right\}$ converges towards the point $h(y)$ in contradiction with the definition of $\left\{D_{n}^{\prime}\right\}$.

After that we assume that there are reflections in the groups $F$ and $F^{\prime}$. Let $E_{0}$ be the closure of a component of $p^{-1}(S \backslash \partial S)$ where $p: E^{\mathbf{1}} \backslash$ $L(F) \rightarrow S$ is the canonical projection. Clearly $E_{0}$ is a hyperbolically convex, closed Jordan domain. We may assume that there is a point $y \in E_{0} \cap S^{1}$ since otherwise $S$ would be compact and this case was treated in $[4]$.

Next we define $E_{0}^{\prime}$ which is a closure of a component of $p^{\prime-1}\left(S^{\prime} \backslash \partial S^{\prime}\right)$ when $p^{\prime}: E^{1} \backslash L\left(F^{\prime}\right) \rightarrow S^{\prime}$ is the canonical projection. Let $L$ be a (closed) hyperbolic line with the endpoint $y$ such that a neighbourhood of $y$ in $L$ is contained in $\operatorname{int} E_{0} \cup\{y\}$. Let $L^{\prime}$ be $L$ transformed by $h$. Then $E_{0}^{\prime}$ shall be the closure of a component of $p^{\prime-1}\left(S^{\prime} \backslash \partial S^{\prime}\right)$ for which a neigh- 
bourhood of $h(y)$ in $L^{\prime}$ is contained in int $E_{0}^{\prime} \cup\{h(y)\}$. It is clear that $E_{0}^{\prime}$ is uniquely determined regardless of the choice of $y$ or $L$.

Suppose then that $T \in F$ is a reflection for which $\operatorname{Ax}(T) \backslash E_{0}$ is a line segment. We claim that $\operatorname{Ax}(\varphi(T)) \cap E_{0}^{\prime}$ is also a line segment. This is obvious if there is a point $y \in \operatorname{Ax}(T) \cap E_{0} \cap S^{1}$. If $\operatorname{Ax}(T) \cap E_{0}$ $\subset E$ we choose a point $y \in E_{0} \cap S^{1}$ and denote by $L$ the hyperbolic line (closed) that joins the points $y$ and $T(y)$. Then if $L$ is not the axis of a reflection of $F$, a neighbourhood of $y$ in $L$ is contained in int $E_{0} \cup\{y\}$ and $L$ does not intersect in $E$ with other axes of reflections of $F$ than $T$. This proves our claim in this case. And it is not difficult to modify the argument if $L$ is the axis of a reflection of $F$.

We need the following fact. If $T$ and $S$ are reflections of $F$ such that the intersection of $\operatorname{Ax}(T) \cap E_{0} \cap E$ with $\operatorname{Ax}(S) \cap E_{0} \cap E$ is a point then the same is true of $\operatorname{Ax}(\varphi(T)) \cap E_{0}^{\prime} \cap E$ and $\operatorname{Ax}(\varphi(S)) \cap$ $E_{0}^{\prime} \cap E$ and conversely. For if this is not the case we could find three reflections of $F$ such that their axes bound a hyperbolic triangle in $E$ and this is impossible since $S$ was assumed to be non-compact.

Thus we have the Jordan domains $E_{0}$ and $E_{0}^{\prime}$. Let $F_{0}$ be the subgroup of $F$ leaving $E_{0}$ fixed. This is equivalent with the fact that $F_{0}$ leaves bd $E_{0}$ invariant. So $F_{0}^{\prime}=\varphi\left(F_{0}\right)$ is the subgroup leaving $E_{0}^{\prime}$ invariant. Next, we define a homeomorphism $h^{\prime}: \operatorname{bd} E_{0} \rightarrow$ bd $E_{0}^{\prime}$ such that

$$
h^{\prime}(T(x))=\varphi(T)\left(h^{\prime}(x)\right) \text { for } T \in F_{0} \text { and } x \in \mathrm{bd} E_{0} .
$$

We set $h^{\prime}: E_{0} \cap S^{1}=h \mid E_{0} \cap S^{1}$. Let $T$ be a reflection of $F$ such that $E_{0} \cap \operatorname{Ax}(T)$ consists of more than one point. Then $\mathrm{E}_{0} \cap \operatorname{Ax}(T)$ is a (closed) hyperbolic segment if there are two reflections $T_{1}$ and $T_{2}$ such that $\operatorname{Ax}\left(T_{i}\right) \cap E_{0}$ is homeomorphic to a line segment and that $\operatorname{Ax}(T) \cap \operatorname{Ax}\left(T_{i}\right)$ is an endpoint of $\operatorname{Ax}(T) \cap E_{0}, i=1,2$, that lies in $E$. If there is only one such reflection $\operatorname{Ax}(T) \cap E_{0}$ is a hyperbolic ray (closed) and if there are not such reflections of $F$ at all $E_{0} \cap \operatorname{Ax}(T)$ is a hyperbolic line (closed). Thus we can always define a homeomorphism $f_{T}: E_{0} \cap \operatorname{Ax}(T) \rightarrow E_{0}^{\prime} \cap \operatorname{Ax}(\varphi(T))$ in such a way that any two mappings coincide on common points and with $h^{\prime}: E_{0} \cap S^{1}$. Besides it is clear that we may suppose

$$
f_{T}(S(x))=\varphi(S)\left(f_{S^{-1} T S}(x)\right) \text { for } x \in \operatorname{Ax}\left(S^{-1} T S\right) \cap E_{0}, S \in F_{0} .
$$

Thus together these mappings $f_{T}$ and $h \mid E_{0} \cap S^{1}$ define a homeomorphism satisfying (4). Then we can by the first part of this proof extend it to a homeomorphism $f^{\prime}: E_{0} \rightarrow E_{0}^{\prime}$ such that (4) holds for all $T \in F_{0}$ and $x \in E_{0}$.

By proposition 2.4 (or by its corollary 2.4.1 which, however, does not take account of the boundary $\left.p^{-1}(\partial S \backslash B)\right)\left(S_{F}, B_{F}, n_{F}\right)$ is equivalent with 
$\left(\left(E_{0} \backslash L\left(F_{0}\right)\right) / F_{0},\left(\left(E_{0} \cap S^{1}\right) \backslash L\left(F_{0}\right)\right) / F_{0}, n\right)$ where $n(x)$ is determined by the stabilizer of $y, p(y)=x$, and a similar result is valid for $\left(S_{F^{\prime}}, B_{F^{\prime}}\right.$, $\left.n_{F^{\prime}}\right)$. Thus we can by corollary 2.4 .3 find a lifting $f$ of the homeomorphism $f^{\prime \prime}:\left(S_{F}, B_{F}, n_{F}\right) \rightarrow\left(S_{F^{\prime}}, B_{F^{\prime}}, n_{F^{\prime}}\right)$ defined by $f^{\prime}$ in the quotient set $\left(E_{0} \backslash L\left(F_{0}\right)\right) / F_{0}$. Then $f$ is a homeomorphism $E^{1} \backslash L(F) \rightarrow E^{1} \backslash L\left(F^{\prime}\right)$ and it can be chosen so that

$$
\begin{gathered}
f(T(x))=\varphi^{\prime}(T)(f(x)) \text { for all } x \in E^{1} \backslash L(F) \text { and } T \in F, \\
f\left|E_{0} \backslash L(F)=f^{\prime}\right| E_{0} \backslash L(F)
\end{gathered}
$$

where $\varphi^{\prime}$ is an isomorphism $F \rightarrow F^{\prime}$. By lemma 3 it can be extended to the whole closed unit disk so that (5) holds for all $x \in E^{1}$ and $T \in F$. It is clear that $\varphi\left|F_{0}=\varphi^{\prime}\right| F_{0}$ and that $\varphi(T)=\varphi^{\prime}(T)$ for such reflections $T \in F$ for which $\operatorname{Ax}(T) \cap E_{0}$ is a line segment. Since these generate $F$, we have $\varphi=\varphi^{\prime}$. This proves the theorem. In proving it we have also proved the fact that a group whose fundamental domain is non-compact and does not contain reflections can be formed as a free combination of cyclic groups. This is perhaps worth noting as a proposition.

Proposition. Let $F$ be a discrete group of the unit disk such that $E / F$ is non-compact and that $F$ does not contain reflections. Then $F$ has a fundamental domain $D$ bounded by hyperbolic lines equivalent in pairs and by hyperbolic rays equivalent in pairs so that if $P$ is such a ray, $P=T(Q)$ where $Q$ is another ray bounding $D$ and $T$ is an elliptic transformation of $F$ with a fixed point that is the common endpoint of $P$ and $Q$. Let $P_{i}$ and $Q_{i}, i \in I$, be the hyperbolic lines or rays bounding $D$ so that $P_{i}=$ $T_{i}\left(Q_{i}\right)$ where $T_{i} \in F$. Let $F_{i}$ be the cyclic group generated by $T_{i}$. Then $F$ is the free product of the groups $F_{i}, i \in I$.

A corollary of theorem 3 is the following.

Corollary. Let $F$ and $F^{\prime}$ be discrete groups of the unit disk such that $F$ contains a free subgroup with two generators, i.e. the limit set of $F$ contains more than two points. Let $\varphi: F \rightarrow F^{\prime}$ be an isomorphism. Then it is geometric if and only if it satisfies the axis condition (2) p. 3 .

Proof: By lemma 3.4 in [4] (2) is necessary. It is also sufficient. For by lemma 3.4 and theorem 2 above we can assume that $F$ and $F^{\prime}$ are groups of the first kind. The conclusion follows now by theorem 3 , since the boundary homeomorphism exists by proposition 3.5.

\author{
University of Helsinki
}

Institute of Mathematics 


\section{References}

[1] Ahlfors, L. Y. - Sario, L.: Riemann Surfaces - Princeton U. P. 1960.

[2] Macbeath, A. M.: The classification of non-Euclidean plane crystallographic groups - Can. J. Math. 6 (1967).

[3] Marden, A.: Isomorphisms between Fuchsian groups (unpublished).

[4] Tukia, P.: On discrete groups of the unit disk and their isomorphisms - Ann. Acad. Sci. Fenn. A. I. 504 (1972).

[5] Zieschang, H. - Vogt, E. - Coldeway, H. D.: Flächen und ebene diskontinuierliche Gruppen - Lecture Notes in Mathematics 122, Springer Verlag, Berlin-Heirdelberg-New York (1970). 